\title{
Gastrointestinal Choriocarcinoma: Case Report
}

Okiro P.0. ${ }^{1} \mathrm{MBChB}$, Oigara R.0. ${ }^{2} \mathrm{MBChB}$, MMed, Ndonga A.K. ${ }^{2} \mathrm{MBChB}$, MMed, Gachii A.K. ${ }^{1} \mathrm{MBChB}$, MMed (Path). 1-Aga Khan University Hospital Nairobi, Department of Anatomic Pathology, 2-St. Mary's Mission Hospital Nairobi. Correspondence: Patricia 0. Okiro, Department of Pathology, Aga Khan University Hospital, P.0. Box 30270, 00100, Nairobi, Kenya. E-mail: patokiro@ yahoo.com

\section{Summary}

Extragonadal choriocarcinomas are rare tumours which are difficult to diagnose. Choriocarcinomas involving multiple sites of the gastrointestinal tract have been rarely reported. We report a case of a 23 year old Para $1+1$ female patient presenting with anaemia and malaena stools for several months. Endoscopy revealed lesions in the stomach, ileum and colon which were found to be choriocarcinomas. Serum $\beta$-hCG levels were also markedly raised. An index of suspicion and a multidisciplinary approach involving the clinical, radiological and pathology teams should aid in arriving at the right diagnosis.Serum $\beta$-hCG is an inexpensive non-invasive test that greatly assists this diagnosis.

\section{Introduction}

Choriocarcinoma arises from the chorionic epithelium of placentas in association with hydatidiform mole, recent abortion or full term pregnancy (1). It is a rapidly growing, highly invasive and widely metastasizing tumour that produces human chorionic gonadotropin (hCG). Choriocarcinomas may also arise from ectopic pregnancies, the gonads, or midline sites, such as the mediastinum, retroperitoneum, and pineal gland, occurring as germ cell tumours (2). Only occasionally have primary neoplasms been reported in parenchymal organs.

Primary extragonadal choriocarcinomas present with unusual clinical features and as such are difficult to diagnose (3).The pathogenesis of these tumours is still uncertain. Extragonadal sites that have been reported include lung, liver, breast, prostate, urinary bladder, nose and gastrointestinal tract (4).

We report a case of multiple gastrointestinal tumours that showed features of pure choriocarcinoma.

\section{Case Report}

A 23 year old Para 1+1 female patient, whose last delivery was in the year 2005, and who had suffered a spontaneous abortion in 2006, was admitted at the Kenyatta National Hospital, Nairobi with complaints of headache, dizziness, lower abdominal pain, fainting, palpitations, easy fatigability and one episode of menorrhagia. The symptoms had been present for 6 months. Her haemogram at that time showed a Haemoglobin level of $4.7 \mathrm{~g} / \mathrm{dL}$, red blood cell count of $2.01 \mathrm{X} 1012$ /L; mean haemoglobin concentration (MHC) $23.4 \mathrm{pg}$, mean corpuscular volume 78.4fL and white cell count 7 X 109/L. She received two units of whole blood and was commenced on haematinics.

Two weeks after the initial presentation she was reviewed at St. Mary's Mission Hospital with severe anaemia and malaena stools. She had negative history of vaginal bleeding at that time. The differential diagnoses considered at the time were peptic ulcer disease and uterine fibroids. Upper gastrointestinal tract endoscopy performed showed a polyp on the anterior gastric wall with evidence of recent bleeding. The polyp was excised and sent to the Aga Khan University Hospital, Nairobi laboratory for histological evaluation

On gross examination, the polyp measured $2 \times 2 \mathrm{cms}$ and had areas of haemorrhage. Microscopic examination revealed tumour infiltrating the mucosa and submucosa that appeared poorly differentiated, with cells showing abundant eosinophilic cytoplasm, pleomorphic nuclei and numerous mitotic figures. The tumour was reported as an infiltrating, poorly differentiated malignant tumour whose origin and histogenesis were not evident from the histological features.

At the patient's review three weeks later, the malaena still persisted. A total gastrointestinal endoscopy was scheduled and performed, which revealed two tumours, one jejunal and the other in the ascending colon. She subsequently had a laporotomy, with short segment bowel resection of the tumours and anastomoses performed. The 


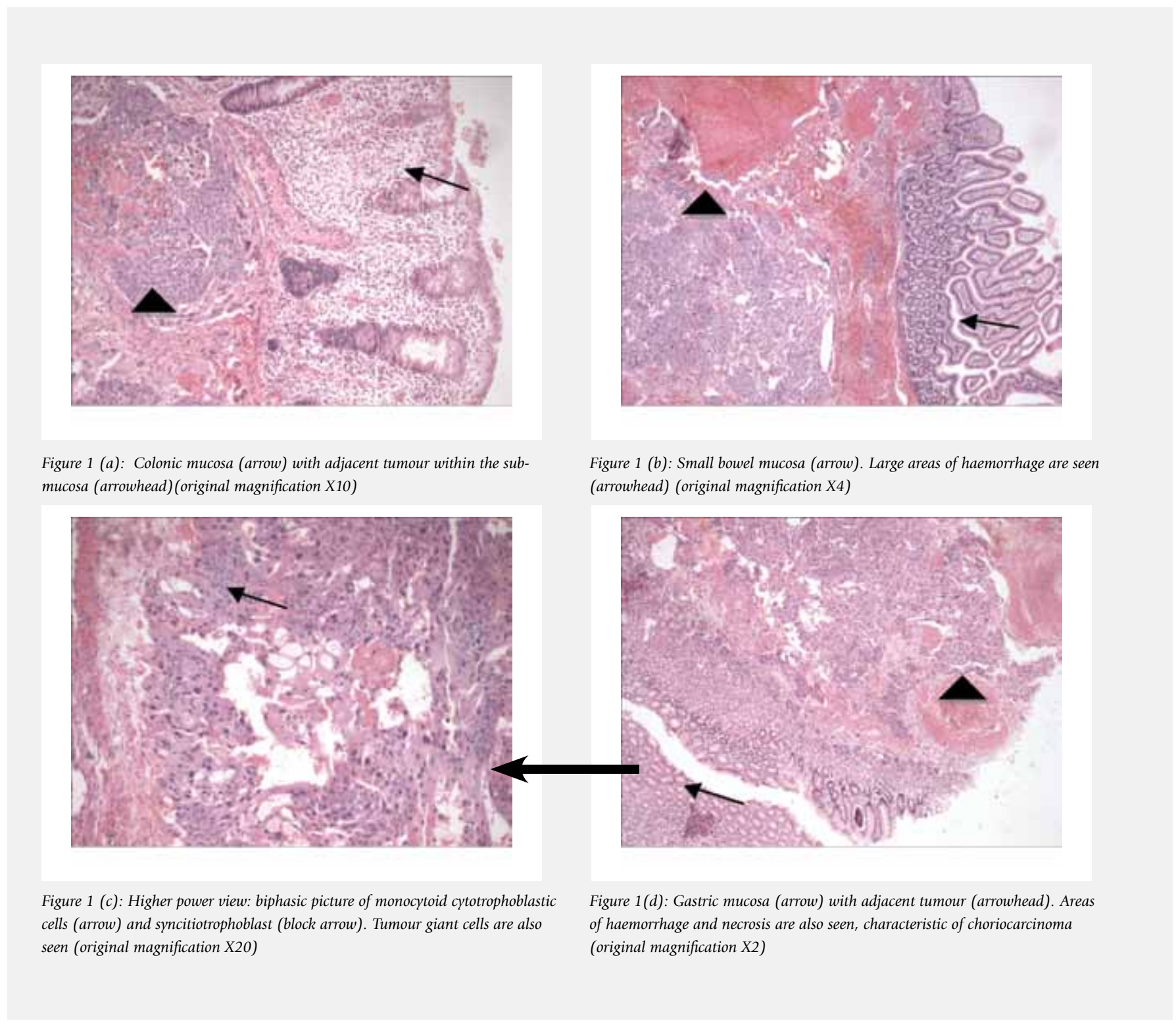

resection specimens were sent to the same laboratory for histological evaluation. On gross examination, both segments showed mucosal ulcers measuring $1.2 \mathrm{cms}$ and $0.5 \mathrm{cms}$ respectively, with raised heaped edges and haemorrhagic floors. Representative sections processed for microscopic evaluation showed ulcerated tumour involving the mucosa, sub mucosa and muscular layer, a biphasic picture of malignant trophoblastic elements, in an intensely haemorrhagic and necrotic background. (Figures $1 \mathrm{a}$ to $\mathrm{d}$ )

The clinicians were appraised of the findings suggestive of choriocarcinoma, and requested to evaluate serum hCG. The initial measure was 504,220mIU/mL.

The slides from the gastric polyp were retrieved and re- viewed (Fig. 1d) and found to be identical to those of the colonic and ileal tumours. The final histopathological diagnosis was indicated as choriocarcinoma, possibly metastatic to the GIT.

Subsequent screening investigations included a chest Xray which was clear, and a pelvic ultrasound which did not reveal any evidence of a gonadal or gestational lesion.

The patient was started on chemotherapy with methotrexate, cyclophoshamide, and Actinomycin D. She received 6 courses of chemotherapy. Her hCG levels were $5,750 \mathrm{mIU} / \mathrm{mL}$ after the first course, and dropped to $46.8 \mathrm{mIU} / \mathrm{mL}$ following the 6 courses. Eighteen months after her initial presentation, serum hCG level was 0 . The 
Gastrointestinal Choriocarcinoma: Case Report

Okiro P.O., Oigara R.O., Ndonga A.K., Gachii A.K.

patient is currently 6 months pregnant with a normal intrauterine gestation.

\section{Discussion}

The term choriocarcinoma generally refers to gestational choriocarcinoma, occurring in the background of complete hydatidiform mole (with an incidence of 1 to $2 \%$ ) (4), spontaneous abortion, ectopic pregnancy or normal delivery (1). Untreated, choriocarcinoma is the most aggressive form of gestational trophoblastic disease.

Choriocarcinomas arising in other organs have been described, several within or along with adenocarcinomas of the organ in question $(2,5)$, and few as pure primary choriocarcinomas. Primary extragonadal choriocarcinomas are rare tumours, and a difficult diagnosis to make in an unusual presentation (3). The pathogenesis of these tumours is still uncertain. Extragonadal sites that have been reported include lung, liver, breast, prostate, urinary bladder, nose and gastrointestinal tract.

The mechanism of onset of non-gestational germ cell tumours has been a subject of ongoing debate. Three hypotheses have been postulated, particularly described for testicular choriocarcinoma (6): i) metastatic tumour with spontaneous regression of the primary tumour, ii) tumours arising from abnormal migration and retention of primordial germ cells during embryogenesis and iii) original non-trophoblastic neoplasm that is transformed into choriocarcinoma

In the gastrointestinal tract, the clinical presentation is similar to peptic ulcer disease or even gastric adenocarcinoma, with haemorrhage. Endoscopy allows access to the tumour in order to permit histological evaluation. However, small biopsies confound the diagnostic process, particularly of pure choriocarcinomas. Kobayashi et al reported that only $8 \%$ of choriocarcinoma cases were diagnosed correctly by biopsy (7).

Early diagnosis, prompt therapy initiation, and sequentatial hCG quantitation are invaluable to favourable outcomes in these patients. Serum hCG is an inexpensive, non-invasive and widely available test that greatly assists in making this diagnosis, as well as in evaluation of patient response to treatment. However this should be considered along with histological features, because other tumours including melanomas, other ovarian and testicular germ cell tumours, malignant lymphoma, and carcinomas of the oesophagus, stomach, pancreas, kidney, liver, lung, urinary bladder, uterus, adrenal gland, breast and other sites can also show increased levels of hCG (4). A total hCG result of more than 100,000 $\mathrm{mIU} / \mathrm{mL}$ in the absence of normal pregnancy strongly suggests gestational trophoblastic disease (GTD) (8). Serial measurement of hCG levels is standard follow-up of women diagnosed with gestational trophoblastic disease. The level of hCG correlates with tumour mass (9). Other diagnostic modalities used in the diagnosis of choriocarcinoma include immunohistochemistry for $\beta$-hCG, cytokeratin (7) and placental alkaline phosphatase (PLAP) (10), human placental lactogen (hPL), SP1 and CEA (4). Molecular genetic studies in primary choriocarcinoma have shown aberrations of adenocarcinomas specific to the locale, as well as of gestational choriocarcinoma (11), suggesting an alternative differentiation pathway for these adenocarcinomas.

Cytogenetic studies may also be useful, using locus-specific minisatellite probes to identify restriction fragment length polymorphisms (RFLPs) in DNA from tumor tissue. These studies help to distinguish gestational from nongestational (germ cell) choriocarcinoma, as well as in documenting the tumor derivation from a preceding molar pregnancy. This is by establishing the androgenetic nature of the tumour (12). For this case, none of these ancillary studies were available.

This case demonstrates a rare presentation of this tumour, with multiple lesions in the GIT, a finding that is rarer still. A high index of suspicion and a multidisciplinary approach should aid in arriving at the right diagnosis. The clinical, histological, and biochemical criteria for a diagnosis of gastrointestinal choriocarcinoma, as well as the patient's response to choriocarcinoma chemotherapy also bears credence to the diagnosis.

\section{References}

1 Olsen JH, Mellemkjaer L, Gridley G et al. Molar pregnancy and risk of cancer in women and their male partners. Am J Obstet Gynecl. 1999; 181: 630-4

2 Jindrak K, Bochetto JF, Alpert LI. Primary gastric choriocarcinoma: case report with review of world literature. Hum Pathol. 1976; 7(5): 595-604

3 Shariat SF, Duchene D, Kabbani W et al. Gastrointestinal hemorrhage as first manifestation of metastatic testicular tumor. Urology. 2005; 66 (6): 1319

4 Rosai J. Pregnancy, trophoblastic disease, and placenta. In: Rosai J, editor. Rosai and Ackerman's Surgical Pathology. 9th ed. Philadelphia, USA: Elsevier; 2006. p. 1737-61. 
5 Hartz PH, Ramirez CA. Coexistence of carcinoma and chorioepithelioma in the stomach of a young man. Cancer 1853; 6(2): 3109-26

6 Yokoi K, Tanaka N, Furukawa K, et al. Male choriocarcinoma with metastasis to the jejunum: a case report and review of the literature. J Nippon Med Sch. 2008 Apr;75(2):116-21.

7 Kobayashi A, Hasebe T, Endo Y, et al. Primary gastric choriocarcinoma: two case reports and a pooled analysis of 53 cases. Gastric Cancer.2005;8(3):178-85.

8 Menczer J, Modan M, Serr DM. Prospective follow-up of patients with hydatidiform mole. Obstet Gynecol. 1980;55(3):346-9.

9 Elliott MM, Kardana A, Lustbader JW, et al. Carbohydrate and peptide structure of the alpha- and beta-subunits of human chorionic gonadotropin from normal and aberrant pregnancy and choriocarcinoma. Endocrine. 1997;7(1):1532.

10 Verbeek W, Schulten HJ, Sperling M, et al. Rectal `adenocarcinoma with choriocarcinomatous differentiation: clinical and genetic aspects. Hum Pathol. 2004;35(11):1427-30

11 Liu AY, Chan WY, Ng EK et al. Gastric choriocarcinoma shows characteristics of adenocarcinoma and gestational choriocarcinoma: comparative genomic hybridization and fluorescence in situ hybridization study. Diagn Mol Pathol. 2001; 10 (3): 161-5

12 Fisher RA, Newlands ES, Jeffreys AJ, et al. Gestational and nongestational trophoblastic tumors distinguished by DNA analysis. Cancer. 1992 Feb 1;69(3):839-45. 